\title{
PLASMA INSTALLATION FOR $\mathrm{TIO}_{2}$ FINE POWDER PRODUCTION: MATHEMATICAL SIMULATION
}

\author{
Frolov V.*, Ivanov D., Shibaev M. \\ Peter the Great St. Petersburg Polytechnic University, Polytekhnicheskaya Str. 29, 195251, Saint Petersburg, \\ Russian Federation \\ * frolov. eed@gmail.com
}

\begin{abstract}
.
The scheme of an evaporator of $\mathrm{TiO}_{2}$ powder based on a plasma torch of combined type is presented. That scheme is characterized by reducing of the equipment cost by decreasing of RF power in comparison to application of usual ICP torches. An analysis of thermo-physical processes of plasma generation as well as heating of fine $\mathrm{TiO}_{2}$ particles is presented. The results were a basis for creating of equipment for the production of fine $\mathrm{TiO}_{2}$ materials and nanostructured $\mathrm{TiO}_{2}$ materials with lower radio frequency $(\mathrm{RF})$ power consumption.
\end{abstract}

Keywords: fine powder production, inductively coupled plasma torch, arc plasma torch.

\section{1. Introduction}

By now a large number of technologies of fine powder and nanopowder production were developed. All existing methods of fine powder and nanopowder production can be divided into two groups: mechanical methods and physical and chemical methods.

To produce fine powders and nanopowders by a mechanical way various types of mills are used in which grinding of material is achieved due to abrasion and impacts 11. The disadvantages of that technique include a possibility of pollution of powder by abrade materials as well as a difficulty of obtaining of powders with a narrow particle size distribution.

Physical and chemical methods of fine powders and nanopowders production are the followings: a vacuum deposition, a sol-method, plasma methods, a laser ablation, etc [2]. In the majority of these methods the initial material is evaporated, moved to the place of deposition and then it is condensed in the form of fine particles and nanoparticles due to intense forced cooling by quenching gas (the exception is the solmethod in which the powder is formed by a chemical reaction of the two solutions). In some cases, the desired product is formed by chemical reactions in the gas phase while the starting reagents are common and readily available materials.

A plasma synthesis of nanoparticles is a very promising method allowing to achieve a high efficiency of the process and a precise control of parameters of the final product [3, 4].

In plasma process the evaporation of initial material and chemical reactions (if required) occur in the thermal plasma. High temperature allows all the initial materials to transit into gaseous state and their subsequent reactions and products condensation. The desired dispersion of produced particles is achieved by controlling of the cooling rate of plasma flow in which a process of condensation from the gas phase occurs.
To produce thermal plasma the industry mainly uses two types of electrical discharges: an arc and a radio frequency (RF) inductively coupled plasma (ICP). Applied to the production of nanopowders the ICP torches have higher technological features compared to the arc plasma torches such as: a generation of plasma in a large volume; a purity of plasma due to the lack of electrodes, simple design; an ability to wide change of a velocity of plasma flow; a simplicity of materials feeding into a plasma stream; a long residence time of material in the plasma jet; a long service life; reliability; an ability of operation with any gas environment, including corrosive one; scalable up to a power of about $10 \mathrm{MW}$.

A disadvantage of application of RF plasma is a necessity of using of quite expensive RF power supplies. Moreover, the cost of those power supplies is significantly increased with increasing of RF power. A combined plasma torch was proposed in order to reduce the cost of equipment by decreasing of $\mathrm{RF}$ power. In that kind of plasma torch an existence of inductively coupled plasma and its ignition is provided by a DC arc plasma torch [5].

The article describes the plasma process of $\mathrm{TiO}_{2}$ fine powder and nanopowder production on the basis of the combined plasma torch as well as analysis of thermo-physical processes of the plasma generation, motion and heating of fine $\mathrm{TiO}_{2}$ particles in plasma.

\section{2. Experimental installation}

Figure 11 represents a scheme of a technological process of $\mathrm{TiO}_{2}$ fine powder and nanopowder production. Plasma created by a plasma torch is used to heat the initial powder. Powder is fed to the outlet of the plasma torch (this method of powder feeding does not affect the generation of plasma inside the torch). Being inside the plasma jet the powder is heated and is completely vaporized (goes into the gaseous phase) 


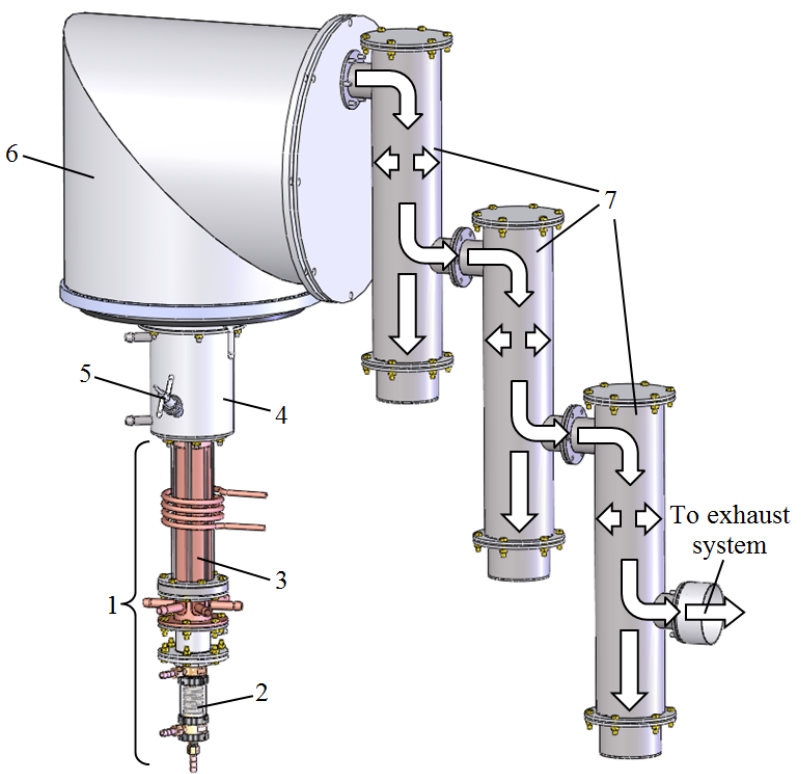

Figure 1. Scheme of the technological process of fine powder and nanopowder materials production: 1 combined plasma torch; $2-D C$ part of the combined plasma torch; 3 - RF part of the combined plasma torch; 4 - evaporating chamber (reactor); 5 - powder feeding tube; 6 - condensing chamber; 7 - trap modules

in the zone of the evaporator. It is well known that nanopowders can be obtained by a condensation of vapor phase during the quenching [6]. Therefore a mixture of plasma gas and evaporated material passes through a nozzle of small diameter wherein it is cooled by cold gas flow.

The resulting fine powder containing nano-sized fraction is collected in a special device namely a trap having a modular structure. The principle of operation of the trap is based on the mechanism of the powder condensation and its deposition on the watercooled walls. A design of the trap module provides the existence of the zone of stagnant gas that lead to increase the deposition rate of powder. The largest fractions of powder are deposited in the first trap module, the smaller ones are deposited in the following ones. Awaited fractions of powder in the trap modules are the followings: more than 1 micron in the first module, from $100 \mathrm{~nm}$ to 1 microns in the second one, less than $100 \mathrm{~nm}$ in the third one. Depending on the mode of operation, the device can produce both amorphous and photocatalytic $\mathrm{TiO}_{2}$. A quantity of trap modules can be changed depending on the powder feed rate.

The above-described features of ICP torch are valid for the combined plasma torch, they are undoubted advantages when the combined plasma torch is used for the synthesis of fine powders and nanopowders.

Large plasma volume allows to realize its uniform loading by the initial material. Slow plasma flow leads to big residence time in plasma (compared to the plasma flow generated by arc plasma torches), so that the initial material are better treated and the final product is more uniform in quality. In addition, high temperature and large plasma volume allow to produce fine powders and nanopowders with high efficiency, and produced powders have a narrow size distribution.

The plasma torch PN-V1 produced by a company "Association Polyplasma" 7] with a channel diameter of $12 \mathrm{~mm}$ was chosen as the basic design of the arc plasma torch [8 10]. It was decided to increase the diameter of the channel and the nozzle of the plasma torch up to $20 \mathrm{~mm}$. The selected diameter will provide a low velocity of plasma so that a steady laminar operation of plasma jet exists at sufficiently high flow rates of plasma gas. Geometry of developed ICP torch (with a diameter of $56 \mathrm{~mm}$ ) was chosen based on preliminary experiments.

The design features of the combined plasma torch namely a distance between an output end of the arc plasma torch and the inductor coils was initial data for the design of the arc plasma torch. Their consideration shows that the length of generated jet of the arc plasma torch should be in the range of $250-350 \mathrm{~mm}$ to provide a reliable ionization of a zone of generation of inductively coupled plasma (i.e. inductor zone).

Thus, the foregoing suggests that the plasma process of fine powder and nanopowder production using a combined plasma torch includes the following steps:

- Generating of plasma in the combined plasma torch (in arc part and ICP part);

- Feeding of a fine powder into the plasma jet and its evaporation;

- Cooling of the vapor phase and condensing of material in the form of nanoparticles.

\section{3. Mathematical model}

For a better understanding of the processes taking place in the combined plasma torch and in the zone of plasma jet mathematical modelling of these processes was carried out.

The simulation was performed using the software ANSYS Fluent 13.0. A 2D axisymmetric case was considered. By the software it was possible to solve simultaneously the following equations: energy equation, continuity equation, motion equation.

The ability to apply user-defined functions (UDF) allowed to introduce into the simulation an electromagnetic problem and non-linear thermophysical properties of plasma (for the sake of simplicity we used pure Ar at $1 \mathrm{~atm})$. Two separate simulations were obtained, for the arc part of the plasma torch and for the ICP part of the torch, and simultaneous modelling of arc part and ICP part of the combined plasma torch. The computational domain was divided by mesh onto about 300000 cells. Boundary conditions for each of the required values are defined at each boundary of the computational domain using simple physical considerations. 


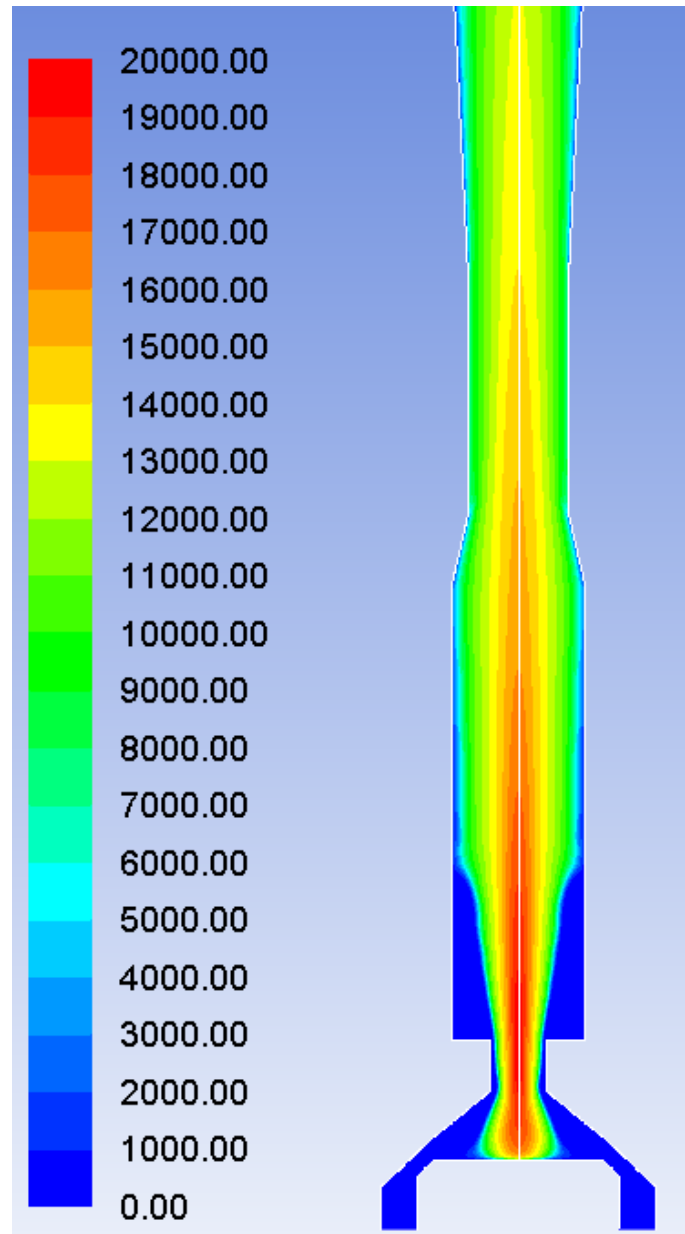

Figure 2. Distribution of plasma temperature in arc plasma torch (arc voltage is $U=70 \mathrm{~V}$, power in plasma is $P=14.5 \mathrm{~kW}$, argon flow rate is $G=25.9 \mathrm{slpm}$ )

The conditions of laminar flow of the arc plasma torch were created to obtain the required length of the arc plasma jet [11]. Arc plasma power was changed in the range of $10-15 \mathrm{~kW}$, argon flow rate was in the range of $15-37 \mathrm{slpm}$.

\section{4. Results and discussion}

The results of the calculation are shown in Figure 2 that presents the distributions of plasma temperature of the arc plasma torch at a power of $14.5 \mathrm{~kW}$ and an argon flow rate of $25.9 \mathrm{slpm}$. In this case the plasma velocity not exceeds $10 \mathrm{~m} / \mathrm{s}$ and plasma temperature not exceeds $3000 \mathrm{~K}$ in the inductor zone of the ICP torch.

In order to select an operation mode of the ICP torch providing the power of plasma jet that is sufficient to vaporize the initial $\mathrm{TiO}_{2}$ powder a series of calculations was carried out in which the power was varied between 20 and $40 \mathrm{~kW}$, the gas flow rate was between 18.5 and $148 \mathrm{slpm}$, the coil current frequency was 1.76 and $5.28 \mathrm{MHz}$ (values from standard range of frequencies in Russian Federation).

Results of a calculation of the ICP part of the combined plasma torch are shown in Figure 3 for

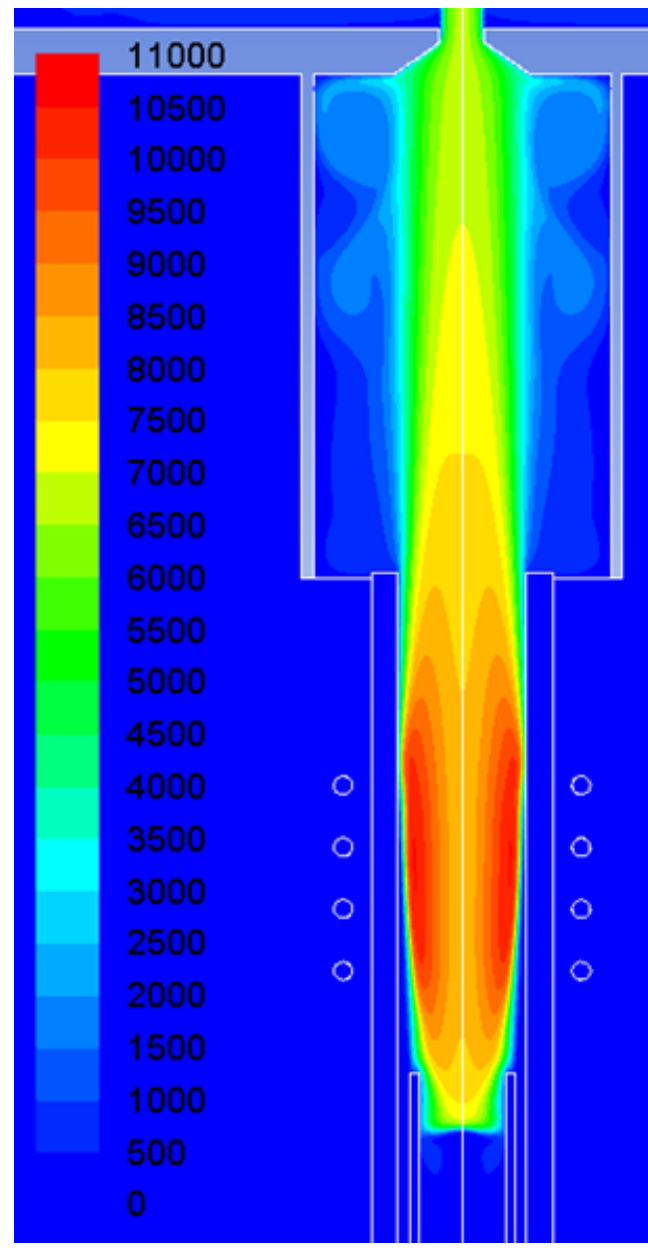

Figure 3. Distribution of plasma temperature in ICP torch ( $R F$ power is $P=30 \mathrm{~kW}$, coil current frequency is $f=5.28 \mathrm{MHz}$, argon flow rate is $G=100 \mathrm{slpm}$ )

operation at a power of $30 \mathrm{~kW}$ and a gas flow rate of 100 slpm.

Calculation showed that a power of the plasma jet (a power at the outlet of the plasma torch) is about $20 \mathrm{~kW}$ in that operation mode. It is most effectively provided at a frequency of $5.28 \mathrm{MHz}$.

The maximum temperature is situated in the zone of energy dissipation. The plasma temperature decreases to 7-8 thousand $\mathrm{K}$ at the exit of the plasma torch.

The results obtained in the previous step (namely the distributions of plasma temperature and velocity at the plasma torch outlet) were used as input data for calculation of plasma jet loaded by initial fine powder. Calculations were carried out using the software ANSYS Fluent 13.0 in 3D setting. This is due to the fact that the powder feeding carried out radially using special water-cooled tube that disturbs an axial symmetry of the geometry.

Results of simultaneous modelling of arc part and ICP part of the combined plasma torch are shown in Figure 4 .

A series of calculations of plasma jet at different powder feed rate $(2-5 \mathrm{~kg} / \mathrm{h})$ and various start velocities $(5-15 \mathrm{~m} / \mathrm{s})$ was carried out $[12$. 


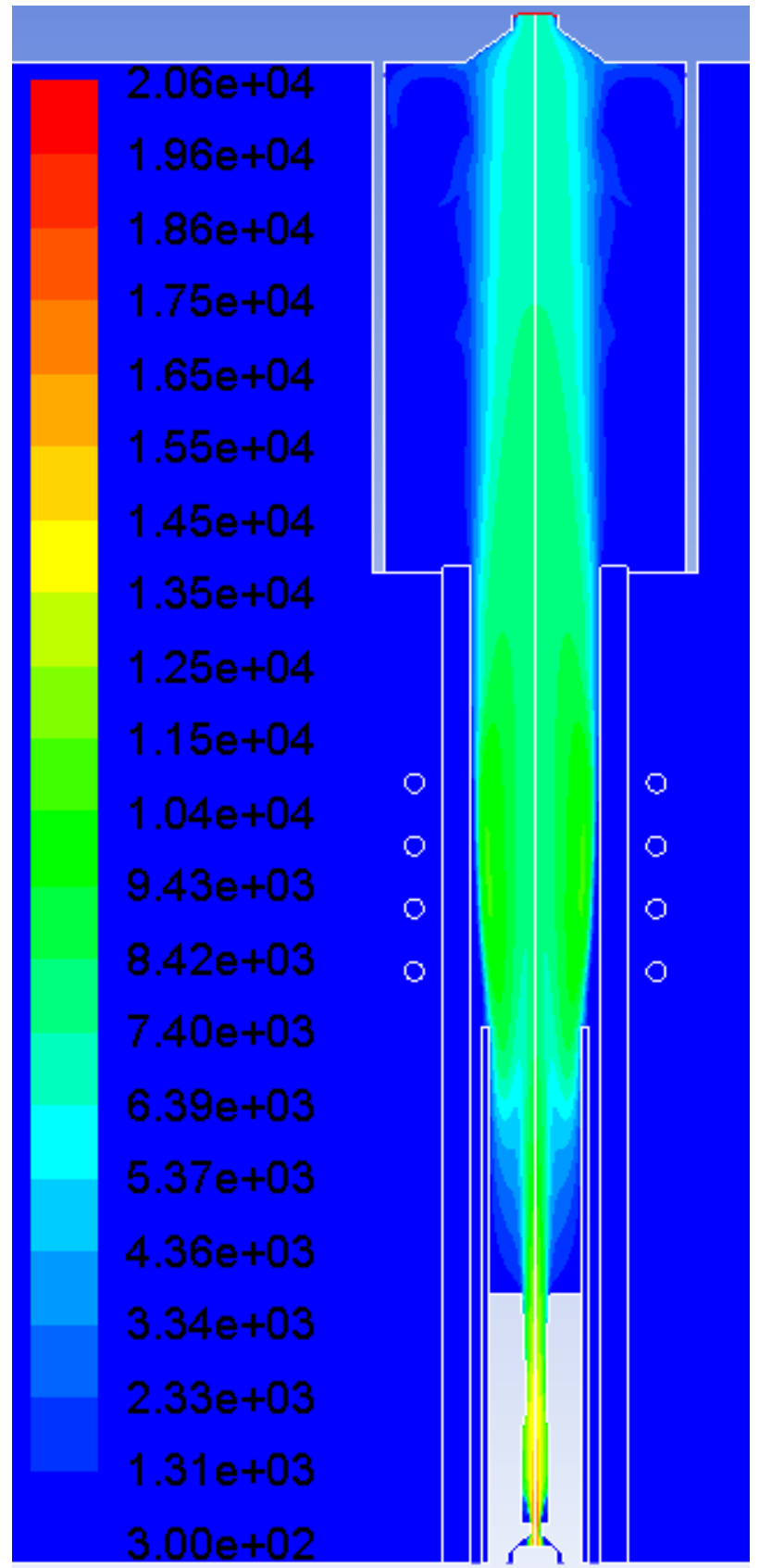

Figure 4. Distribution of plasma temperature in combined plasma torch

Initial powder was $\mathrm{TiO}_{2}$ with a mean diameter of $15 \mu \mathrm{m}$.

Best results (optimal trajectory of particles and its heating) were obtained at a powder feed rate of $4 \mathrm{~kg} / \mathrm{h}$ and a start velocity of $10 \mathrm{~m} / \mathrm{s}$ (see Figure 5 ).

Evaporation of particles of the initial powder was not taken into account for the presented calculations.

However, this aspect will be taken into consideration in our future work. Evaporation will result in a change of the plasma composition and thereby to change its properties. The calculation of such plasma composition shows that plasma in the presence of vapours of titanium is more uniform, with a higher thermal conductivity (due to greater influence of electrons)

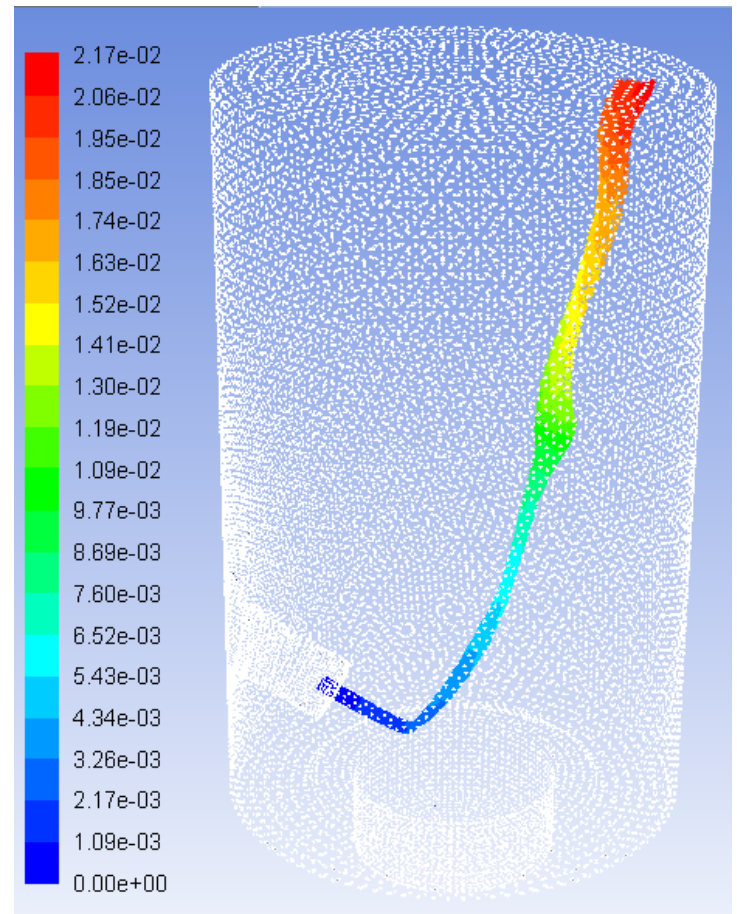

Figure 5. Trajectory and residence time (in s) of $\mathrm{TiO}_{2}$ particles with a mean diameter of $15 \mu \mathrm{m}$ (powder feeding rate is $4 \mathrm{~kg} / \mathrm{h}$, start velocity is $10 \mathrm{~m} / \mathrm{s}$ ) [12]

which will increase the efficiency of the evaporation of the powder.

The process of cooling of gas flow containing vapour and condensation of fine powder also expected to consider in next steps using the model described in [13.

\section{5. Conclusion}

The new scheme of plasma process of fine $\mathrm{TiO}_{2}$ powder production based on a combined plasma torch is proposed. That scheme is characterized by reducing of the equipment cost by decreasing of RF power in comparison to to application of usual ICP torches.

An analysis of thermo-physical processes of plasma generation as well as heating of fine $\mathrm{TiO}_{2}$ particles is presented. The results were a basis for creating of equipment for the production of fine and nanostructured $\mathrm{TiO}_{2}$ materials with increased efficiency and lower power consumption.

Based on these investigations a combined plasma torch for fine powder and nanomaterials production was developed. That torch consists of series-connected two parts: the arc plasma torch and the ICP torch.

ICP torch has the following parameters: the frequency is $5.28 \mathrm{MHz}$, the inner diameter of the torch is $56 \mathrm{~mm}$, the plasma power is $30 \mathrm{~kW}$ and the gas flow rate is $74.0-110.0 \mathrm{slpm}$.

A patent [14] was obtained on the base of this investigation. 


\section{References}

[1] C. C. Koch. Synthesis of nanostructured materials by mechanical milling: Problems and opportunities. Nanostruct. Mater., 9(1):13-22, 1997.

[2] R. E. Kirk D. F. Othmer and A. Seidel. Chemical Technology. 5th edn. Wiley, Hoboken, NJ, 2004.

[3] R. M. Young and E. Pfender. Generation and behavior of fine particles in thermal plasmas - a review. Plasma Chem. Plasma Process., 5(1):1-37, 1985.

[4] Boulos M.I. Jurewicz J.W. Nessim C.A.A.M. Plasma synthesis of metal oxide nanopowder and apparatus therefor. US Patent 6,994,837, 2006.

[5] V. Frolov et al. Experimental investigations of the hybrid plasma torch with reverse vortex stabilization. Romanian Journal In Physics, 56(Suppl.):36-40, 2011.

[6] M. I. Boulos P. Fauchais and E. Pfender. Thermal Plasmas: Fundamentals and Applications. New York: Plenum, 1994.

[7] Association Polyplasma. Official cite. http://www.polyplasma.spb.ru/.

[8] V.Ya. Frolov M.V. Dubov B.A. Ushin. Analysis of thermal behavior of plasma torches. Nauchnotehnicheskie vedomosti SPbGPU, 78:125-129, 2009.

[9] V. Frolov et al. Research and development of plasma technologies of spraying of coatings. Proc. 18th Symposium on Physics of Switching Arc. Editors: V.Aubrecht and M.Bartlova, Brno, 162-165 (2009).

[10] M. Baeva et al. Proc. XIX International Conference on Gas Discharges and their applications; ed. Prof. Z. C. Guan, Beijing, 158-161 (2012).

[11] W. Pan W. Zhang W. Zhang C. Wu. Generation of long, laminar plasma jets at atmospheric pressure and effects of flow turbulence. Plasma Chem. Plasma Proc., 21(1):23-35, 2001.

[12] M.A. Shibaev. Study of combined plasma torch for plasma technology of nano titanium dioxide production. Master Thesis. SPbSPU, St. Petersburg (2012) (in Russian).

[13] N. Gonzalez M. El Morsli P. Proulx. Production of nanoparticles in thermal plasmas: A model including evaporation, nucleation, condensation, and fractal aggregation. J. therm. spray techn., 17(4):533-550, 2008.

[14] A.V. Lopota V.Ya. Frolov D.V. Ivanov. Combined plasma torch. RF Patent 2440701, filed August 6, 2010, and issued January 20, 2012. 